to deter doctors from using the valuable technique of exchange transfusion in the severely anaemic patient in need of blood.-I am, etc.,

Pembury Hospital,
Tunbridge Wells,

D. G. Daniel.

Kent.

REFERENCES

1 Fullerton, W. T., and Turner, A. G., Lancet, 2 Ward, T., Brit. med. F., 1952, 1, 631.

\section{Indomethacin and Peptic Ulcer}

SIR,-Last year we reported a high incidence of peptic ulcers (5 out of 18 patients) in patients with rheumatic diseases treated with indomethacin (11 January 1964, p. 118). Since September 1963 we have used only capsules of indomethacin, and we now wish to report a much-lessened incidence of gastro-intestinal side-effects.

Fourteen patients with a variety of rheumatic diseases were treated for periods varying from one to 17 months with capsules containing indomethacin, $25 \mathrm{mg}$., thrice daily with food. All patients had a barium-meal examination before the treatment and at least once during the period of treatment. Three patients who gave a history

of peptic ulcer received concomitant anticholinergic-drug therapy and antacids.

One patient died during the course of treatment from cerebral haemorrhage, but at necropsy there was no ulceration of the gastro-intestinal tract. One of the patients with a past history of peptic ulcer developed a gastric ulcer; indomethacin was discontinued and the occult-blood test became negative. The second patient with a history of peptic ulcer who had developed ulceration with indomethacin tablets did not develop an ulcer when given indomethacin capsules. The third patient with a peptic ulcer history did not develop ulceration either on tablets or on capsules.

Thus the incidence of peptic ulceration can be reduced by switching from tablets to capsules. Moreover, other side-effects such as vertigo, headache, nausea-which were relatively common in patients treated by tablets -did not occur. In our previous report we suggested that a lower dosage of indomethacin would overcome troublesome side-effects, and we feel that this has been achieved without any reduction of anti-rheumatic efficacy.We are, etc.,

\section{Medical Dept. I, Stockholm K, Swe \\ Olle Lövgren. Erik Allander.}

\section{Better Method for Occluding Umbilical Cord}

SIR,-Mr. C. K. Vartan has recently described (10 October, p. 944) the use of a rubber band cut from latex intravenous tubing for " tying" the umbilical cord. Like him, I have found this method most satisfactory and started using it for the same reason-that the ordinary cord tie, being through the band, which is autoclaved in. a small paper envelope as part of the large package of instruments for the delivery.

At delivery, using the tape as a hold, the closed jaws of the artery forceps are thrust through the centre of the rubber band. The cord is clamped with these artery forceps

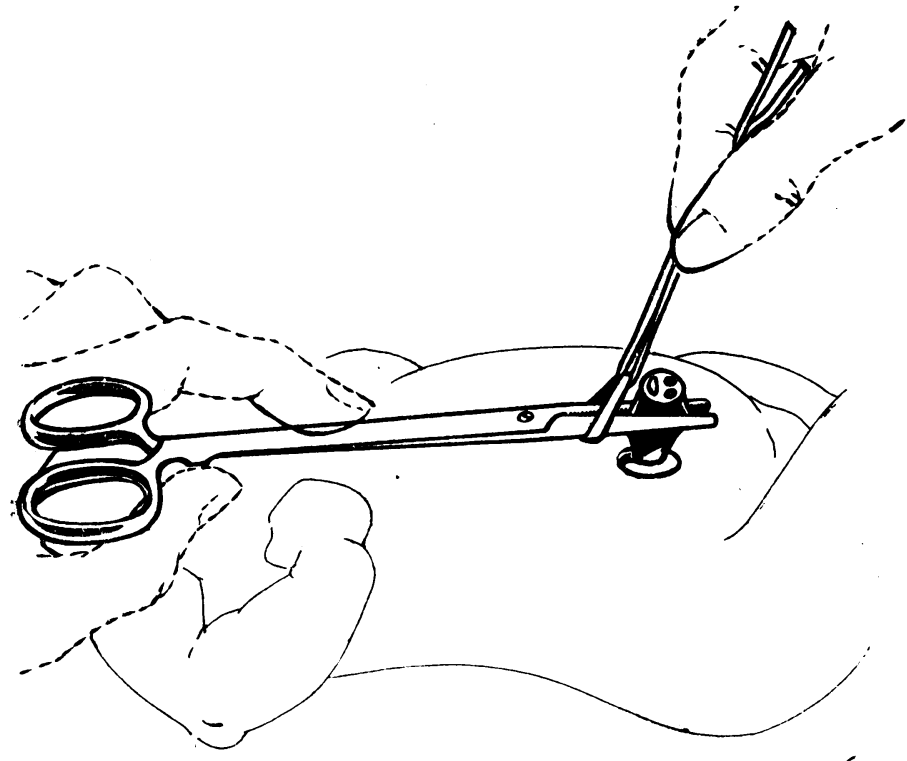

non-elastic, will not take up as the cord shrinks. Repeated cord tying is tedious and not a complete guarantee against haemorrhage. An artery forceps left on the cord is safer, but the instrument is remarkably awkward in a baby's cot, and may exert considerable leverage on the umbilicus.

The method of application of the rubber band, as shown in the drawing, is simpler than that described by Mr. Vartan. The rubber bands (two to three $\mathrm{mm}$. in diameter), are cut from the latex intravenous tubing with one cut of a large pair of scissors. A piece of umbilical-cord tape is threaded about 1 in. $(2.5 \mathrm{~cm}$.) from the abdomen. Another forceps is then applied on the placental side, in the usual way, and the cord divided. The rubber band is pulled over the cord stump, as shown in the diagram. With the tape the rubber band can be readily positioned about half an inch from the skin edge. The tape is then pulled out. It has been the practice to leave the artery forceps on for one hour, although this is doubtless unnecessary. The sliding of the band down the cord empties it of blood, which is desirable.

In an unsuccessful attempt to simplify the method even further the rubber band was then put on the artery forceps before the intrument was autoclaved. This proved unsatisfactory, as the laiex band lost some of its elastic character through heating in contact with the metal. Autoclaving the bands on their own does not affect their elasticity.

In one year's use of this method no haemorrhage has occurred from the cord The cord separates in the normal length of time.-I am, etc.,

Palmerston North

New Zealand.

John Crowley.

\section{The Catarrhal Child}

SIR,-Dr. D. H. Garrow in his article on “The Catarrhal Child" (20 March, p. 773) is justified in condemning the operation of T's and A's as a cure for all catarrhal ailments in children. All E.N.T. consultants would agree that to-day they will only perform this operation if there are very clear indications for it.

His remarks about deafness in children must be challenged as they are at variance with the facts. He says that " deafness after acute otitis media is usually short lived and clears up spontaneously" and he recommends energetic treatment with penicillin. Whilst it is true to say that many children with acute otitis media are treated by penicillin it is fact that this is very frequently oral penicillin, which is prescribed by the general practioner in vast quantities. The condition of "glue ear" or secretory otitis media has become extremely common in recent years and there is no really satisfactory treatment for it once it has become established-the child may be condemned to a permanent conductive deafness. Recent experience has tended to show that the child who has had his adenoids removed is less likely to develop this condition. It is also possible that oral penicillin is a factor in the production of it.

I was speaking at a symposium on deafness recently and I was surprised to find that the extreme prevalence of this disease is not appreciated by many paediatricians; nor do many realize what a serious degree of conductive deafness it can produce. At least $4 \%$ of our population have a considerable degree of hearing loss and a much larger proportion have slight degrees of deafness ; much of this deafness is conductive and can presumably be prevented. We should appreciate that by refusing to remove the tonsils and adenoids of children who get recurrent attacks of infection and by treating them with penicillin we may be adding to the already considerable amount of conductive deafness which is present to-day.-I am, etc.,

Doncaster, Yorks.

P. H. BEales,

\section{Hormones and Hair}

SIR,-I am induced by your leading article "Hair Cycles and Hair Patterns" (6 March, p. 604) and Dr. Arthur J. Rook's most excellent lecture on "Endocrine Influences on Hair Growth" (6 March, p. 609) to report my own clinical experiences using transplantation homologous punch-grafting and oestrogen in treating male baldness and generalized thinning of hair in the menopausal female.

Dr. Rook states that grafting experiments convincingly demonstrate donor dominance. The success of transplantation homologous 